\title{
ON MULTIPLY TRANSITIVE PERMUTATION GROUPS
}

\author{
G. P. MONRO and D. E. TAYLOR
}

(Received 24 August 1977)

Communicated by W. D. Wallis

\begin{abstract}
We present a direct combinatorial proof of the characterization of the degree of transivity of a finite permutation group in terms of the Bell numbers.
\end{abstract}

Subject classification (Amer. Math. Soc. (MOS) 1970): 20 B 20.

The $k$ th Bell number $B_{k}$ is the number of partitions of a set of $k$ elements (Comtet, 1974, p. 210). It has been observed by Merris and Pierce (1971) that if $G$ is a group of permutations of a set $X$, then $G$ is $k$-fold transitive if and only if

$$
\frac{1}{|G|} \sum_{g \in G} \pi(g)^{k}=B_{k}
$$

where $\pi(g)$ denotes the number of elements of $X$ fixed by $g$. The proof given by Merris and Pierce is by induction on $k$ and uses the recurrence relation $B_{k+1}=\sum_{j=0}^{k}\left(\begin{array}{l}k \\ j\end{array}\right) B_{j}$. In this note we give a proof based directly on the interpretation of $B_{k}$ as the number of partitions of a set of $k$ elements.

THEOREM. $G$ is $k$-fold transitive if and only if

$$
\frac{1}{|G|} \sum_{g \in G} \pi(g)^{k}=B_{k} \text {. }
$$

Proof. Let $Y$ be the set of $k$-tuples of elements of $X$, and let $G$ act on $Y$ by setting $g\left(\left\langle x_{1}, \ldots, x_{k}\right\rangle\right)=\left\langle g\left(x_{1}\right), \ldots, g\left(x_{k}\right)\right\rangle$. Note that if $g \in G, g$ fixes $\pi(g)^{k}$ elements of $Y$. It follows from a theorem of Burnside on the number of orbits of a permutation group (Huppert, 1968, p. 536) that if $Y$ has $N$ orbits under $G$, then

$$
\frac{1}{|G|} \sum_{\sigma \in G} \pi(g)^{k}=N
$$

Now, let $\mathscr{P}$ be a partition of the set $\{1, \ldots, k\}$. Let $Y_{\mathscr{g}}$ be the subset of $Y$ consisting of those $k$-tuples $\left\langle x_{1}, \ldots, x_{k}\right\rangle$ such that $x_{i}=x_{j}$ if and only if $i$ and $j$ are both in the same block of $\mathscr{P}$. Clearly $Y_{\mathscr{g}}$ is a union of orbits. If $G$ is $k$-fold transitive each $Y_{\mathscr{g}}$ 
is in fact an orbit, so there are $B_{k}$ orbits. If $G$ is not $k$-fold transitive, consider $\mathscr{P}_{0}=\{\{1\}, \ldots,\{k\}\} . \quad Y_{\mathscr{g}_{0}}$ will be the union of more than one orbit, so altogether there will be more than $B_{k}$ orbits.

Weaker forms of this theorem are discussed by Huppert (1968, p. 599) and van Lint (1974, p. 31).

\section{References}

L. Comtet (1974), Advanced Combinatorics (Reidel, Dordrecht, 1974).

B. Huppert (1968), Endliche Gruppen I (Springer-Verlag, Berlin, 1968).

R. Merris and S. Pierce (1971), "The Bell numbers and $r$-fold transitivity", J. Combinatorial Theory (A) 12, 155-157.

J. van Lint (1974), Combinatorial Theory Seminar, Eindhoven University of Technology (SpringerVerlag Lecture Notes in Mathematics, 382, Berlin).

Department of Pure Mathematics

University of Sydney

N.S.W. 2006

Australia 